\title{
Cross-layer issues in MAC protocol design for MIMO ad hoc networks
}

Michael A. Jensen

jensen@byu.edu

A. Lee Swindlehurst

Michele Zorzi

James R. Zeidler

Adam L. Anderson

See next page for additional authors

Follow this and additional works at: https://scholarsarchive.byu.edu/facpub

Part of the Electrical and Computer Engineering Commons

\section{Original Publication Citation}

Zorzi, M., et al. "Cross-Layer Issues in MAC Protocol Design for MIMO Ad Hoc Networks." Wireless Communications, IEEE 13.4 (26): 62-76

\section{BYU ScholarsArchive Citation}

Jensen, Michael A.; Swindlehurst, A. Lee; Zorzi, Michele; Zeidler, James R.; Anderson, Adam L.; Rao, Bhaskar; Proakis, John; and Krishnamurthy, Srikanth, "Cross-layer issues in MAC protocol design for MIMO ad hoc networks" (2006). Faculty Publications. 302.

https://scholarsarchive.byu.edu/facpub/302 


\section{Authors}

Michael A. Jensen, A. Lee Swindlehurst, Michele Zorzi, James R. Zeidler, Adam L. Anderson, Bhaskar Rao, John Proakis, and Srikanth Krishnamurthy 


\title{
Cross-LaYer ISSUES IN MAC Protocol DESIGN FOR MIMO AD HOC NETWORKS
}

\author{
Michele ZORZI, UNIVERSITY OF PADOVA, ITALY, AND \\ UNIVERSITY OF CALIFORNIA AT SAN DIEGO \\ James Zeidler, Adam Anderson, Bhaskar RaO, AND John Proakis \\ UNIVERSITY OF CALIFORNIA AT SAN DIEGO \\ A. Lee SWIndlehURSt ANd Michael Jensen, Brigham Young UNIVERSITY \\ SRIKANTH KRISHNAMURTHY, UNIVERSITY OF CALIFORNIA AT RIVERSIDE
}

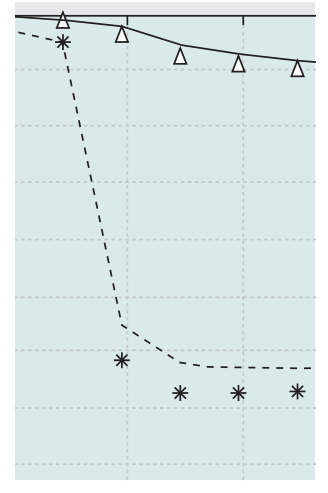

The benefits of using multiple antennas in wireless communications have been

known for some time. However, there are many open issues regarding how this increased capacity at the physical layer can be exploited to provide improved networking performance by the use of new protocol design techniques.

\section{ABSTRACT}

In this article, we discuss research and design issues that arise in the development of networking protocols for an ad hoc network where nodes are equipped with multiple antenna elements and have the ability to perform signal processing operations as required by adaptive beamforming, interference cancellation, and space-time coding.

\section{INTRODUCTION}

The benefits of using multiple antennas in wireless communications have been known for some time [1-3]. In particular, Foschini [3] showed that the use of multiple antennas at both the receiver and the transmitter makes it possible to significantly increase the link capacity, effectively making use of multiple parallel radio channels in the same band, by separation in space. Following that seminal paper, many researchers have contributed to this area, but there remain many open research issues regarding how this increased capacity at the physical layer (PHY) can be exploited to provide improved networking performance by the use of new protocol design techniques.

The field of ad hoc networks has enjoyed significant interest in the research community in recent years, as the capability of setting up a self-organizing wireless network on demand is attractive in many application scenarios, including disaster relief, temporary events, and battlefield situations [4]. Recent capacity results on ad hoc networks [5] suggest that in very large deployments the available throughput per node may be limited, as it is a decreasing function of the number of nodes in the network. While the exact relationship depends on specific assumptions made on the transmitter properties and on the traffic characteristics, the result is believed to hold under fairly general conditions. Since the level of interference in the network is correlated with the number of nodes transmitting simultaneously, it is important to look for ways to mitigate the interference and to improve the transport capacity of such networks. The use of smart antennas and multiple-input multiple-output (MIMO) technology is a promising PHY approach to boost the network throughput, thanks to the exploitation of the spatial dimension and the resulting interference reduction; however, achieving its full benefits requires a thorough understanding of the physical-layer properties and behavior as well as the networking design criteria and objectives.

In the area of ad hoc networks, there has been some recent interest in understanding how the availability of smart antenna nodes affects both the design and the performance of networking protocols, especially at the medium access control (MAC) and routing layers [6]. There are a number of studies on the use of directional antennas, but this model does not fully address the spatial multiplexing capabilities that might be achieved by MIMO systems. Some recent studies are available in which the authors try to address more general MIMO systems by factoring in more detailed models $[7,8]$, but this is an area which is not yet well understood. In this article we will address the issues involved in exploiting MIMO channels and antenna arrays in improving network operation and protocol performance.

We consider a cross-layer approach, where protocol design is tightly coupled with a deeper understanding of the physical layer and channel behavior. The major focus of this work is the MAC and PHY layers, although some considerations could be made for routing as well. This means that the protocols must be designed in a way that is specifically targeted towards exploiting the availability of multiple antennas and the specific MIMO techniques used (and solving the problems that this availability raises), but it also means that the PHY and MAC must be able to 
exchange measurements and parameter values in order to enable cross-layer optimizations that would not be possible in traditional network architectures. In following a cross-layer approach, it is very important to use caution in order to avoid serious drawbacks, as described in [9]. Therefore, we view the cross-layer design of ad hoc MAC protocols and its full integration with the PHY layer as a very difficult (and largely as yet unsolved) research problem, as is understanding which cross-layer mechanisms are effective and which PHY/MAC quantities and parameters should be exchanged across the layers.

This article is presented in three parts. In the first part, we provide a high-level discussion of what we consider the key technical challenges in this cross-layer approach, and provide some background on MIMO techniques and capabilities from a networking perspective. The second part gives an example of a cross-layer protocol that attempts to exploit $\mathrm{PHY} / \mathrm{MAC}$ interaction and exchange of parameters. This provides insight about how a cross-layer approach can be translated into design strategies and on some of the issues that should be taken into account. As many of these results and considerations depend on the availability of channel state information and on its accuracy, a solid understanding of channel estimation techniques and on their accuracy and validity in time is a key ingredient of the MAC design addressed in this article. For this reason, in the third part of the article, we review some of the recent literature on channel estimation, including some of our own recent contributions. The perspective here also involves trying to understand how this can be helpful at the protocol layers when trying to make connections towards MAC design criteria, different time scales, and so forth.

\section{SOME MOdELING AND DESIGN ISSUES}

In this section we provide a qualitative discussion of some issues that we consider important in modeling and designing simpler and more effective protocols.

\section{SpATIAL Processing}

Before embarking on a discussion of how MIMO technology can impact network performance, it is essential to understand how MIMO systems exploit the channel spatial dimension [10]. Generally speaking, two nodes - each equipped with multiple antennas and communicating over a multipath propagation environment - can theoretically signal over multiple independent and parallel channels or "spatial modes." The number and quality of these modes depends on the propagation characteristics (the number of multipaths along with their gain, departure angle, and arrival angle) and the antenna array topology.

The early studies regarding MIMO PHY technology mostly focused on a point-to-point or single-user link. While this prior work has been valuable in revealing achievable performance bounds and in demonstrating effective signaling and detection strategies, researchers have also recognized the need to address the multi-user case typical of wireless networks. There has been some interesting recent work on this topic [11-13], as well as on the issue of combining MIMO technology with waveforms that are naturally suited for multi-user communications, such as spread spectrum [14] or orthogonal frequency-division multiplexing (OFDM) $[15,16]$. We observe, however, that these multi-user PHY studies have not been widely considered by the networking community when devising MAC schemes.

Naturally, one could use traditional multiaccess schemes (which, for narrowband communications, try to avoid simultaneous transmissions) and exploit MIMO simply to improve the performance of each established link. This approach, however, is suboptimal, since limiting the use of MIMO to increasing the point-to-point throughput does not exploit the MIMO-enabled spatial modes to facilitate parallel transmissions. This is intriguing, given the limitations of common networking strategies. For example, in 802.11, data transmission is preceded by a RTS/CTS exchange that alerts all nodes within reach that they should refrain from transmitting until the data exchange being negotiated is over. However, since MIMO processing allows simultaneous parallel transmissions to (or from) different users, more aggressive schemes in which simultaneous transmissions are encouraged rather than forbidden seem appropriate. In this case, RTS/CTS exchanges can be used by nodes to gain awareness of traffic conditions in the network and to make scheduling decisions about packet transmissions in a distributed way. An example of a protocol designed following these principles is presented in this article.

\section{MIMO PHY ALGORITHMS}

When the nodes of a wireless network possess multiple antennas, the extra degrees of freedom may be used in several ways, as outlined in the paragraphs below. Note that these advantages are not mutually exclusive; they are achieved by essentially the same means, and the degrees of freedom provided by the antennas may be "distributed" to simultaneously realize gains in all of the areas listed below.

Transmit Diversity - The same information (perhaps encoded differently) can be transmitted by more than one antenna, thus increasing the probability that the information is accurately received. A common way of achieving diversity is through space-time coding (STC) [17]. Orthogonal space-time block coding is a popular and very effective STC technique where a block of modulation symbols is transmitted in a different order from each transmit antenna, with appropriate modifications (sign changes, conjugation, etc.) so that the transmitted data block is orthogonal, and decoding is simplified at the receiver. Other STC methods such as space-time trellis and space-time turbo coding have also been proposed. Coding can be employed in the frequency and polarization dimensions as well [18]. The gains promised by the transmit diversity approaches mentioned above rely on the assumption that the channels between each transmit antenna and the receiving node fade indepen-
When the nodes of a wireless network possess multiple antennas, the extra degrees of freedom may be used in several ways. Note that these advantages are not mutually exclusive; they are achieved by essentially the same means. 


Given a perspective
where protocol
design and operation
is to be tightly
coupled with the
PHY and channel
behavior, it is critical
to be able to
understand which
channel parameters
can be estimated
and which are useful
in the proposed
cross-layer approach.

dently. While it is typically assumed that the receiver must have channel state information (CSI) for decoding, knowledge of CSI at the transmitter is not required.

Increased SNR/Range - Multiple antennas can be used to focus the transmission or reception of energy to certain key directions, thus increasing the SNR or range of a wireless link. A typical means of doing this is through linear beamforming [19]. When two communicating nodes are in each other's line-of-sight (LOS), beamforming amounts to choosing antenna weights so that "beams" are pointed in each node's direction. Side lobes cannot be eliminated in such instances, and some interference is produced in other directions, although it can be controlled. Of course, LOS propagation is not required to achieve gains via beamforming. With CSI at the transmitter, the multipath structure of the channel, typically revealed through the singular value decomposition of the channel matrix, can be used to design weights that maximize the link SNR. The resulting "beams" may have no relationship to the LOS direction.

Improved Spectral Efficiency - While SNR gains via beamforming can be used to increase data rates by means of denser signal constellations, multiple antennas can also increase throughput by means of spatial multiplexing, where multiple independent data streams are sent over a single link, and sorted out by means of spatial processing at the receiver. V-BLAST is an example of such a technique, and it only requires CSI at the receiving node [20]. However, capacity-achieving spatial multiplexing requires CSI at the transmitter as well, and amounts to sending symbols using multiple orthogonal transmit beams. The power of each transmitted beam is determined via water-filling in order to achieve capacity [21].

Interference Nulling - The converse of beamforming is often referred to as "null-steering," in which the weights are adjusted to prevent rather than enhance transmission to or reception from certain directions. Interference nulling can occur at either the transmitter, where one desires to reduce the interference seen by other nodes, or at the receiver, where it is desired to eliminate contributions from other active links [22]. As with beamforming, interference nulling does not require LOS propagation, but it does require CSI wherever it is employed, at either the transmitter or the receiver [23].

Multiple Access - The subjects in the three preceding paragraphs are all motivated by scenarios involving point-to-point links, with contributions from other users considered to be interference. Multiple antennas can also be used for multiuser access, either for uplink (multiple nodes transmitting data to a single receiving node) or downlink (a single node transmitting data to multiple receive nodes) communication. It is this additional multiple access capability that could have the most significant impact on the higher layers of an ad hoc wireless network. In situations where the nodes are power constrained, the uplink and downlink cases are not symmetri- cal. The SNR per link in the uplink case will be higher, since in the downlink the transmitting node must divide its power among the nodes it is communicating with. This has important implications for wireless networks. Downlink space-division multiple access (SDMA) also has the disadvantage of requiring CSI at the transmitter and receivers, unless multi-user detection is employed at all of the receivers, and all transmitted data is decoded by everyone. Otherwise, since the receiving nodes cannot cooperate (unlike in the case of the collocated antennas at the receiver for spatial multiplexing above), the transmitter must ensure that data destined for one node do not interfere with the others it is attempting to communicate with $[12,13]$. This is only possible if the transmitter can separate the users spatially, which in turn is only possible if their channels are known. For the uplink case, CSI is only required at the receiver, although transmit CSI could be used to improve SNR by means of beamforming.

As discussed above, and as is explained in more detail below, the ability of nodes to realize the advantages provided by multiple antennas depends on the properties of the communication channels between each pair of nodes and any interfering sources, and the ability of the network nodes to obtain reliable information about these channels. Furthermore, in cases where the transmitter cannot maintain adequately accurate CSI to achieve a desired performance, it is possible to realize a portion of the gains by using channel distribution information (CDI), as discussed in the subsection "Channel Knowledge Time-Scale Issues" below.

\section{Channel Estimation and EFfects of Channel Estimation ERrors}

Given a perspective where protocol design and operation is to be tightly coupled with the PHY and channel behavior, it is critical to be able to understand which channel parameters can be estimated and which are useful in the proposed cross-layer approach. This is a hard problem to solve, since it requires a very deep understanding of both layers as well as the development of new performance studies taking a nontraditional approach.

For a useful cross-layer design approach, it is important to consider how channel conditions and lower-layer designs affect the protocol performance, and how the different time scales involved at the various layers relate to each other. Therefore, from a protocol-design perspective, it is important to understand what are the main channel parameters and the time scales on which they vary, as well as the accuracy with which their values can be provided.

In view of the importance of this topic and its potential implications on MAC protocol design criteria as well as performance, we return to this topic subsequently, and provide more extensive technical results and insights.

\section{Realistic Antenna Models}

As a representative example of the types of studies that need to be performed when using MIMO PHY technology in a multi-user network, consid- 
er the case of beamforming in an LOS environment. In the recent literature on ad hoc networking with directional antennas, purely directional radiation patterns have been mostly considered. One reason for this is that it is relatively easy to study protocols in this case, and it is also relatively easy to adapt existing protocols based on the argument that some interferers will not be seen by a directional node pointing in a different direction, and also that some directional transmissions will only affect nodes within their angle of radiation. The fact that nodes may have different awareness about what happens in different directions leads to some design issues, because of which, adaptation of existing protocols (e.g., IEEE 802.11 for WLANs) is not straightforward, but requires some additional mechanisms [6]. However, it is clear that directional antennas where the gain is constant in a given angle and zero outside do not exist in practice; rather, one has to account for nonconstant gains as well as side lobes which are responsible for the transmission of energy in unwanted directions. These improved models have recently been considered in the networking literature, but are not yet widely used [24]. Similar studies need to be performed in order to understand how other MIMO approaches, such as those outlined in the subsection "MIMO PHY Algorithms" above, will impact networking protocols. The key consideration is to first understand the interference created by a transmission based on the approach under investigation, and then to devise protocol rules based on this information. This remains an open area of research. It is therefore clear that there is a need to identify the various options provided by MIMO networks and multiple antenna systems, and to relate the various techniques to protocol design choices.

\section{RECEIVER VS. TRANSMITTER}

Directional patterns as well as beamforming can be performed at both the transmitter and the receiver. However, there are some fundamental differences that are too often overlooked in protocol design. A fundamental difference between the transmitter and the receiver is that the transmitter is power constrained, whereas the receiver is not. More specifically, the cost for a node to transmit simultaneous streams to two users is about twice the cost of transmitting to a single user, since the major energy consumption component is in fact the transmission power. Conversely, on the receiving side, the cost of receiving is not proportional to the number of beams. In fact, simultaneous reception in multiple modes only requires that the same set of antenna samples (which are taken only once) be processed by DSP in several different ways (the "beamformers"). Thus, the additional cost of using one more mode is the cost of performing one more linear combination on the antenna samples, which is very small in general (e.g., compared with the energy consumption to keep the receiving radio $\mathrm{ON}$ ) and can usually be ignored.

Therefore, the trade-off between direction selectivity and gain (i.e., antenna gain when using a single beam and less or no antenna gain when in omnidirectional mode) only applies to the transmitter, where the total power limit has to be divided among the various beams, whereas it does not exist at the receiver, where omnidirectional reception with directional gain can be achieved by running in parallel as many beamformers as needed to cover the whole angular domain.

\section{MAC SCHEMES FOR MIMO Ad HOC NETWORKS}

Traditional networking research has modeled the physical layer by constructing simplified and, in many cases, unrealistic abstractions that make it easier to perform both analytical and simulation-oriented studies of the protocols developed. The use of high-fidelity models at the PHY tends to slow down simulations and may make analyses intractable. A popular model that is often adopted at the MAC layer is the use of circular ranges with omnidirectional antennas. With this model, it is assumed that nodes within the so called transmission range of a node are able to decode a packet from the node whereas those that are within the sensing range detect the node's transmission but are not able to decode it. With this model, a collision-free reception requires that all the nodes that are within the sensing range of a receiver are precluded from performing transmissions. The circular range model, often called the unit disk graph (UDG) model, also facilitates easy neighbor discovery; all the nodes that are within a given node's transmission range are the node's neighbors. Recent efforts supplement this model by including losses due to shadowing and/or multipath Rayleigh fading within this circular footprint. MAC protocols (such as the IEEE 802.11 MAC) are often designed with this simplified model in mind. This model, which is rather simplistic even in the simpler omnidirectional case, is clearly inadequate for use in a multi-user MIMO setting. The transmission pattern using a multiplicity of antennas is complex and difficult to model. Furthermore, a multiplicity of communicating pairs that are in close proximity to one another could share the available bandwidth simultaneously. Given this, the design of a MAC protocol is challenging and will have to account for physical-layer dependencies.

Complexities in Neighbor Discovery - As described above, a plurality of techniques are possible with multiple-antenna equipped nodes, including the use of space-time codes, interference cancellation techniques, spatial multiplexing, and so forth. In many of these scenarios, CSI is required in order to perform the requisite MIMO processing. At the receiver, CSI is typically obtained by means of training symbols embedded in the transmitted signal. The training data transmission is usually uncoded (equal-gain symbols from each of the transmit antennas) and is not beamformed, since that would imply that the transmitter already has CSI. As a result, the neighboring nodes who hear the training data transmission will often be different from those who might hear a later space-time encoded or beamformed signal from the transmitter once it obtains CSI. Thus, identifying the correct set of reachable
Directional patterns

as well as

beamforming can

be performed at

both the transmitter and the receiver. However, there are some fundamental differences that are too often overlooked

in protocol design. 
Imposing a frame

structure and

ensuring that the

nodes transmit their

CSI at the same

time requires

that nodes be

synchronized.

However, with

mobility,

synchronization may

not be easy to

maintain. neighbors with a MIMO transmission is a challenging and still unsolved issue. This problem becomes considerably simplified if one assumes the aforementioned (unrealistic) directional antenna beam patterns; this has been explored to some extent in the literature $[25,26]$.

Obtaining Channel State Information - As emphasized above, CSI is essential for the full utilization of a multi-antenna channel. High-data-rate multi-user MIMO techniques certainly have merit during data transmission, though it is not so clear whether MIMO techniques are justified (and if so how they should be invoked) during the stage where control messages are sent due to the difficulty of obtaining CSI. One possible scenario would be to use currently available singleantenna techniques for initial signaling handshakes (e.g., RTS/CTS) and insert training at the beginning of the data phase, and full MIMO techniques would only be employed for data transmission. Another option is explored in the next section, where a MAC protocol utilizes CSI and MIMO techniques for RTS/CTS handshakes as well as for data transmission. This requires the nodes and their environment to be static enough that the channel does not change from one link activation to the next.

The availability of CSI feedback will also determine whether an open-loop system or a closed-loop system is to be used. If the channel state is fairly stable, then receiver-based feedback could be invoked to achieve spatial multiplexing or transmit beamforming. If the channel changes fairly rapidly, an open-loop system (e.g., based on the use of space-time codes) may be in order.

An appropriate frame structure must be utilized in the protocol design to enable acquisition of CSI and multi-user packet reception and transmission. An element of frame format construction for ad hoc networking protocols that has received attention is the utilization of a fixed contention window, followed by a data packet [26]. The benefit is that the data window is guaranteed to be collision-free. The popular 802.11 protocols, on the other hand, use a MAC based on CSMA/CA, which does not utilize this format. The best frame format and protocol for an ad hoc network is an open research topic.

Imposing a frame structure and ensuring that the nodes transmit their CSI at the same time requires that nodes be synchronized (an assumption that we make in our design in the next section). However, with mobility, synchronization may not be easy to maintain. If synchronization is lost, then MIMO transmissions and SISO transmissions may be performed simultaneously and the implications have to be carefully considered.

Maintaining Channel State - With mobility, it is clear that the topology of the network changes dynamically. While connectivity will change on a relatively coarse time granularity, channel state changes on a finer granularity. If a node has a large number of neighbors, obtaining and maintaining the CSI with respect to all of these neighbors will be prohibitive in terms of cost. Thus, one might choose to perform topology control wherein a node maintains logical connectivity and updated CSI for only a subset of its neigh- bors. Then, given the aforementioned frame construction, the CSI could be periodically refreshed for these neighbors. While topology-control methods have been explored with the UDG model in mind [27], the problem becomes much more challenging given the complex transmission patterns that result in our scenario of interest.

Trade-offs between Rate, Range, and Reliability - As described above, one could use MIMO for diversity (transmission of the same information on the different antennas) or for spatial multiplexing (or beamforming) [28]. The models that are required from the perspective of the MAC layer differ in the two cases. With diversity, a decrease in BER for the same average SNR is achieved due to the so called diversity gain. At the MAC layer, this decrease could translate into an increase in transmission reliability, an increase in transmission range, an increase in transmission rate (by using a reduced level of forward error correction or a higher level modulation), or a better ability to tolerate interference which, in turn, could translate into a higher number of simultaneous transmissions in the same area. Depending on the performance objective or quality-of-service (QoS) requirement, the MAC layer may appropriately trigger the right functionality at the physical layer. The trade-offs between the different objectives from the perspective of cross-layer design will have to be studied in depth.

From the MAC-layer perspective, one of the main benefits of spatial multiplexing is an increase in rate via a higher number of parallel communication sessions. Given this, the main challenge that arises is to ensure that the number of simultaneous users approaches the limit of what is possible, but does not exceed that limit (so as to prevent collisions or loss of data). In the process, the design has to account for the fact that the neighbors of different nodes differ in an ad hoc networked setting.

Access Methods and Trade-offs - Typically, media access control could be either done as per a deterministic (possibly dynamic) schedule or via random access. Scheduled access is typically contention-free, whereas random access is contention-based.

CSI could be potentially used to determine how packet transmissions may be scheduled. The MAC layer may schedule multiple packet transmissions/receptions on links based on the achievable link SNRs and on their correlations. Depending on the schedule, it may be necessary to categorize nodes (or even antennas) into transmitters and receivers. Schedules have to be formed dynamically; statically scheduling transmissions a priori may not yield the best performance results, as the channel varies dynamically. Furthermore, construction of schedules requires synchronization between nodes (at least in terms of packet transmissions). There has been prior work on scheduled MAC-layer design for MIMO [7]. However, this work does not take into account the physical-layer dependencies and the dynamic-channel variations.

A random access scheme, on the other hand, may result in the suboptimal use of the available capacity if the number of simultaneous transmis- 
sions in a region is higher than what can be accommodated by the antenna elements. Furthermore, prior to any random access transmission, the transmission of training sequences or pilot tones will have to be appropriately stimulated. Most of the previous MAC protocols designed for ad hoc networks are based on a random access strategy defined by the IEEE 802.11 standard. In the following section, we modify this MAC protocol for use with MIMO in some constrained settings.

Opportunistic space-division multiple access (SDMA) has been considered for use with MIMO in cellular networks [29]. While opportunistic SDMA is a feasible approach in cellular networks since the base-station forms beams on the downlink to listening mobile users, in ad hoc networks each node can either be a transmitter or a receiver at a given instance in time. Thus, in order to employ opportunistic SDMA, a node would have to first ensure that the set of neighbors with which it wants to communicate are in the receive mode. A suitable feedback mechanism will also have to be constructed.

\section{AN EXAMPLE OF Cross-LAYER PHY/MAC DESIGN}

In this section we briefly describe a MAC protocol designed to take advantage of a cross-layer approach, and provide some sample results. More details and results can be found in [8].

At the physical layer, we assume that the interference cancellation mechanism proposed in [30] is used, which provides an effective means to receive multiple signals. The MAC protocol is based on a collision avoidance mechanism, where all nodes send RTSs and CTSs before data transmission and conclude with an ACK to confirm correct packet reception. RTS (request to send) messages are used by prospective transmitters to signal their need to send a packet to the intended receivers, who then reply with a CTS (clear to send) if they are able to receive that packet. In networks using omnidirectional antennas, this RTS/CTS exchange is meant to let everyone within reach know about data exchanges so that all these nodes refrain from initiating transmissions, thereby protecting the ongoing data exchange from interference. When transmissions are not omnidirectional, it does not make much sense to block all nodes within reach, as MIMO technology makes it possible to have parallel transmissions in the same area (under proper channel conditions). The role of RTS and CTS is then different in this case, as it is possible for nodes to receive many RTSs simultaneously, and multiple data packets can be cleared for transmission even if they overlap, as separation is achieved in the spatial domain. The RTS/CTS handshake can then be newly interpreted as a means to let nodes know about the overall traffic conditions in the network, so that nodes can decide how many and which transmissions should be cleared via the CTSs. What in omnidirectional networks is a simple binary task (send CTS or not) becomes here a more complex decision process whose rules can be studied and optimized in order to maximize the performance.
In the protocol proposed in [8], packet transmissions are assumed to be synchronized at all nodes: all RTS transmissions start at the same instant, and so do CTS, data, and ACK transmissions, so that the data exchanges are organized in synchronous frames. This can be readily achieved with current technology and allows for correct spatial multiplexing of data and simplified receiver processing for interference cancellation. ${ }^{1}$

Packets in the network are generated according to a Poisson process of parameter $\lambda$ (packets per second per node): each packet is assigned a random destination and a random length of $k \times$ 1000 bits, with $k$ uniformly chosen in the set $\{1$, $2,3,4\}$. Nodes can keep a maximum of 30 packets in their queue. Transmissions take place frame by frame, each frame consisting of an RTS, a CTS, a data packet, and an ACK. Signaling packets are transmitted with a single antenna and are 200 bits long; data packets may be sent with multiple antennas, according to the packet length and using one 1000-bit block (called stream) per antenna. As a first step, in this preliminary study, we only considered uncoded transmissions. Preceding all transmitted packets is a training sequence for channel estimation, one per used tx antenna. We assume that, due to finite processing and memory, nodes can only estimate (using the training sequences) the channel conditions of a limited number of signals: one protocol design issue concerns the strategies to decide which signals should be detected and which should be left as unknown noise (and, as a result, cannot be canceled) [8].

We let any node in the network decide how many antennas to use for transmission depending on the intended receiver's distance. RTSs are then composed by selecting packets from the queue on a FIFO basis, and allocating antennas according to the distance of the intended destination. The RTS contains information about the number of antennas to be used in transmission, so that the receivers can know how many training sequences will be used. A single RTS packet may contain multiple transmission requests. Nodes receiving RTSs try to decode them and then decide which transmissions to allow by sending a CTS, while also considering network load issues. We have developed and studied three different policies as follows.

- NFT (do Not Follow Traffic): each node sends a CTS to clear the streams intended for it, and neither decodes nor cancels any other streams; all transmissions for other nodes are part of the unknown interference term.

- PFT (Partially Follow Traffic): each node first uses training sequences to estimate the channels of all streams meant for it, and then uses the remaining training sequences (if any) to detect and cancel interference coming from other streams.

- FT (Follow Traffic): each node sends a CTS to clear at least one of the streams meant for it, and evaluates other requests in order of decreasing received power: if the evaluated request is meant for the node, it grants it with the CTS, otherwise it simply decodes and cancels the signal in order to enhance the correct decoding probability of the desired streams.
In order to employ

opportunistic SDMA, a node would have to first ensure that the set of neighbors with which it wants to communicate are in the receive mode. A suitable feedback mechanism will also have to be constructed.

\footnotetext{
${ }^{1}$ In the performance analysis, as an approximation and for the sake of simplicity, we assume all transmissions to be symbol-synchronous. This is by no means required for the interference cancellation at the receiver to work properly, so that synchronization is not a critical issue in the proposed scheme.
} 


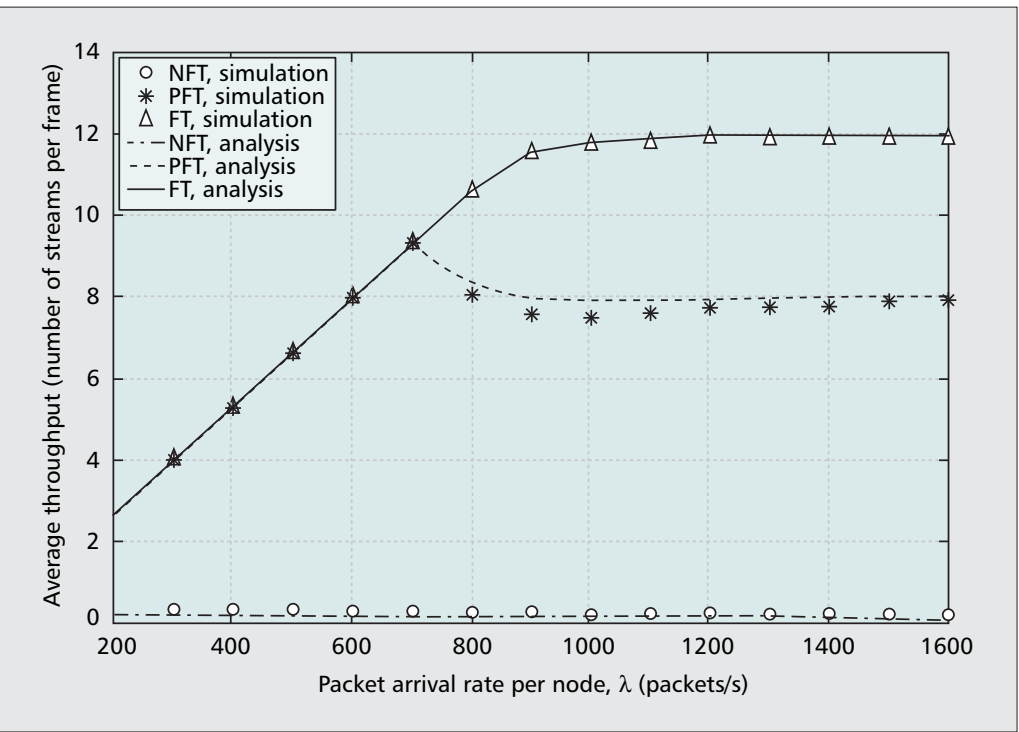

- Figure 1. Average throughput as a function of network load for different CTS policies, analytical and simulation approaches.

Note that in both PFT and FT, each receiver tracks exactly the same number of incoming signals, including intended data streams as well as strong interferers to be canceled. The difference between the two policies is that PFT maximizes the reception of intended traffic (and is therefore more prone to uncanceled interference), whereas FT gives priority to cancellation of strong interferers (thereby somewhat reducing the amount of intended traffic but greatly increasing its reliability). Under any policy, a CTS may respond to multiple RTSs, and this in turn will enable multiple transmissions from different nodes to coexist at the same time. It is worth emphasizing here the inherent cross-layer nature of these strategies, where PHY information about channel strength and accuracy of channel estimation drives protocol decisions at the MAC layer, which in turn defines how the PHY (the use of training sequences and the interference cancellation algorithm at the receiver) should be run.

Nodes then transmit data streams according to the CTSs received, and wait for the ACK. Streams related to the same packet are ACK'd separately, so that only the erroneous streams are retransmitted in the following frame using the appropriate number of antennas, and a packet is finally removed from the transmitter's queue when all of its streams have been correctly received. A backoff mechanism is used, so that nodes which do not receive a CTS in response to an RTS assume the destination to be overloaded, and defer transmissions.

Some example results are given in Figs. 1 and 2 , which report the average throughput, defined as the number of successful streams per frame, and the success rate, or the ratio between successful streams and streams sent. They refer to a fully connected network (all nodes visible to each other) with 25 nodes, each with eight antennas. The channel model includes distance-dependent path loss as well as Rayleigh fading, which is assumed to be uncorrelated for different pairs of transmit-receive antennas. The fully detailed bit-by-bit reception processes, including interference cancellation as well as the MAC protocol operation, have been included in a single simulation program. As can be clearly seen, our trafficaware policies greatly outperform the straightforward application of 802.11 (NFT), showing promising results for increased network efficiency. These results clearly show that the benefit of MIMO technology can be enjoyed only by careful design of the higher-layer protocols, whereas a poor design of the MAC layer leads to unacceptably poor performance, despite the increased PHY potential.

\section{A NOTE ON Simulation Performance Evaluation}

When it comes to evaluating the performance of cross-layer schemes where accurate models of the physical layer are to be used, we encounter several difficulties. First of all, some of the most widely used network simulation tools (e.g., ns-2 [31]) have poor support for physical-layer functionalities, and model the PHY behavior rather questionably, which is a major problem. A significant improvement in this sense would be needed. Another significant difficulty is that, even assuming availability of a simulation tool that allows the incorporation of physical layer details and properly models various aspects such as beamforming or interference cancellation, one would need to perform bit level simulations for proper modeling of PHY behavior, and cover a time span which makes the protocol performance results statistically meaningful. In fact, there is a fundamental mismatch between the time scales involved at the MAC and PHY layers which would potentially result in extremely time-consuming simulations, hampering any serious study of the problem.

Therefore, there is a clear need for simulation models that incorporate proper approximations of the physical layer in order to make it possible to run network simulations with the desired degree of confidence. Unlike the current situation, where network simulators use a drastically simplified PHY model, what we invoke here is the development of analytical approximations for PHY performance that are adequate for network-layer performance evaluations. It is worth noting at this point that one of the reasons these approximations are not readily available is that the degree of accuracy required in this case would probably be seen as unacceptably bad from a strictly PHY point of view, whereas it is possible that even fairly gross approximations may actually do a good job in the overall network evaluation. This step clearly requires collaboration between the two communities, and understanding of each other's needs in order to build effective tools for joint studies. Even though this requires some effort in trying to adapt one's own mindset, we believe this could go a long way in making it possible to effectively study complex systems and to produce efficient cross-layer design approaches.

An example of a possible approach in this direction is reported in [32], where an analytical approximation is proposed for the interference cancellation algorithm and is used in a network 
simulation of the protocol presented in this section. The accuracy of the results is shown in Figs. 1 and 2, where we report both the network simulation results with analytical PHY approximation, and the fully bit-level simulated network performance. As can be seen, the accuracy of the approximation is very good, whereas the simulation time to obtain the results was between 10 and 100 times shorter.

\section{Channel Estimation and KnOWLedge}

In the previous section, we presented an example of a MAC protocol that makes use of PHY and channel information in order to drive the access mechanism, in a cross-layer fashion. A natural question (and a difficult research issue) is how this information can be provided and how it can be used based on its accuracy, scope, validity in time, and so on. In order to make it possible to design and operate cross-layer protocols in a realistic scenario, one must understand the issues related to channel estimation and identify how the related information can be gathered, represented, exchanged, and finally used. While full integration of channel-estimation techniques in the protocol design and operation is well beyond the scope of the present article and will be part of our future work, here we present relevant results that are to be seen as the basis on which to build these cross-layer interactions. In particular, in this section we deal with three main issues: how estimation is performed in MIMO systems, and how accurately; issues in channel estimation in multi-user scenarios; and how long channel estimates can be considered to be valid in various situations of interest.

Typically, CSI is learned (or estimated) at the receiver by observing the channel output that corresponds to the preset training symbols (pilots). The accuracy of these estimates depends on various system parameters such as the pilot SNR, the Doppler spread of the received signal, and the properties of the channel estimation algorithm. In addition, since the channel state information at the transmitter in a frequencydivision duplex (FDD) system has to be sent by the receiver, quantization errors and feedback rate further degrade the accuracy of the CSI at the transmitter. Practical systems also employ channel coding to provide improved performance, and the system performance is also affected by the coding parameters, the decoding algorithm, the interleaving depth, and the Doppler spread. Therefore, there exists a very important trade-off for resource allocation in terms of time slots and power between pilot symbols and data symbols. Furthermore, welldesigned training sequences and pilot structures are also very important to improve the efficiency of the channel estimation [33-36]. In addition, in order to achieve better spectral efficiency than the conventional training-based estimation methods, code-aided joint channel-estimation and data-detection algorithms offer a promising alternative at the expense of computational complexity [37].

In this section we will discuss the factors determining the frame error rate and the bit

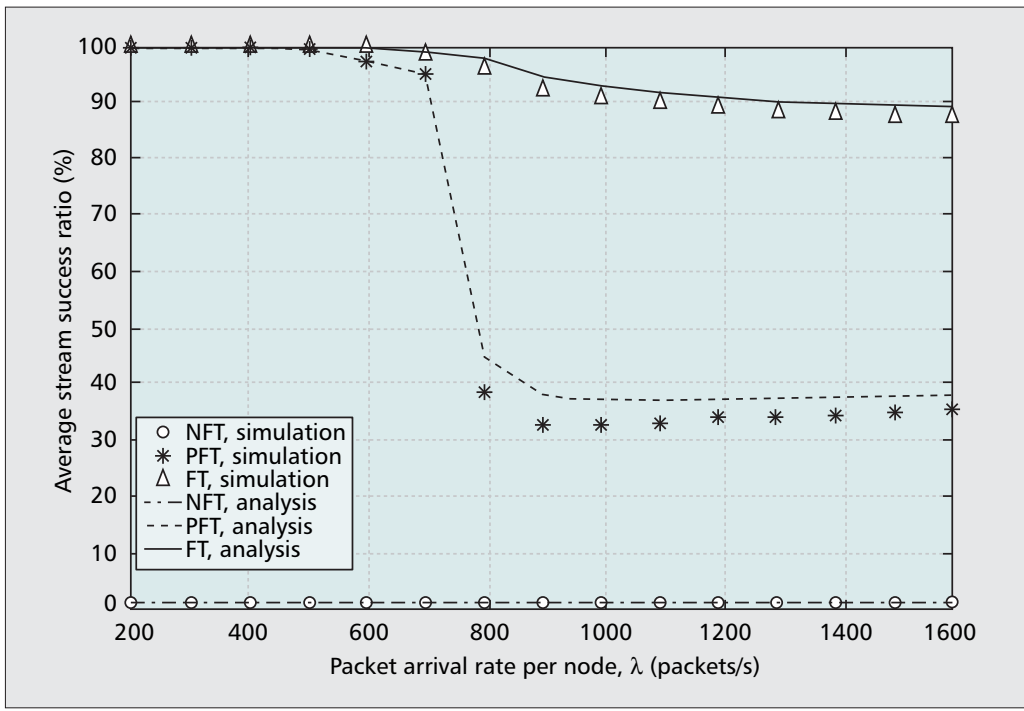

Figure 2. Average ratio of successfully decoded streams to sent streams as a function of network load for different CTS policies, analytical and simulation approaches.

error rate for three transmit diversity schemes: single-input single-output (SISO) and two MIMO formats, Alamouti [38] and closed-loop transmit diversity (CLTD). The MAC layer can optimize the code rate, the coding algorithm, the frame size, and so forth from the predicted error rates for each link and also determine the best link from the available links. The frame-level statistics define the average performance over a frame of data. When the symbol-level parameters are changing much faster than the frame period, the MAC layer may benefit more from knowledge of the frame level statistics. Alternatively, the MAC layer can check the link statistics to continuously optimize the link performance, but with a much higher system overhead. A discussion of the time scale issues in providing MIMO physical-layer data to the MAC layer is discussed below.

Naturally, the appropriate design criteria are dependent on the modulation format and will be different for single-user and multi-user scenarios. The scheduling of users in a multi-user network is one of the fundamental issues addressed at the MAC layer and, consequently, the appropriate protocols will be dependent on the modulation format selected. In this section we summarize some pertinent results on CSI accuracy for three different modulation formats: direct sequence (DS) code-division multiple access (CDMA), frequency hopped (FH) spread spectrum (SS), and OFDM. A substantial body of literature exists for the CSI accuracy of DSCDMA modulation and will be summarized briefly below. DS-CDMA is designed for multiuser networks, but requires accurate power control $[39,40]$ unless advanced receivers are used $[30,41,42]$. However, the improved near-far resistance is achieved under certain assumptions which may not hold in ad hoc networks, especially in a hostile environment. For example, the LMMSE multi-user detector in [41] and the receiver in [30] assume short spreading codes and user synchronization, while the successive 


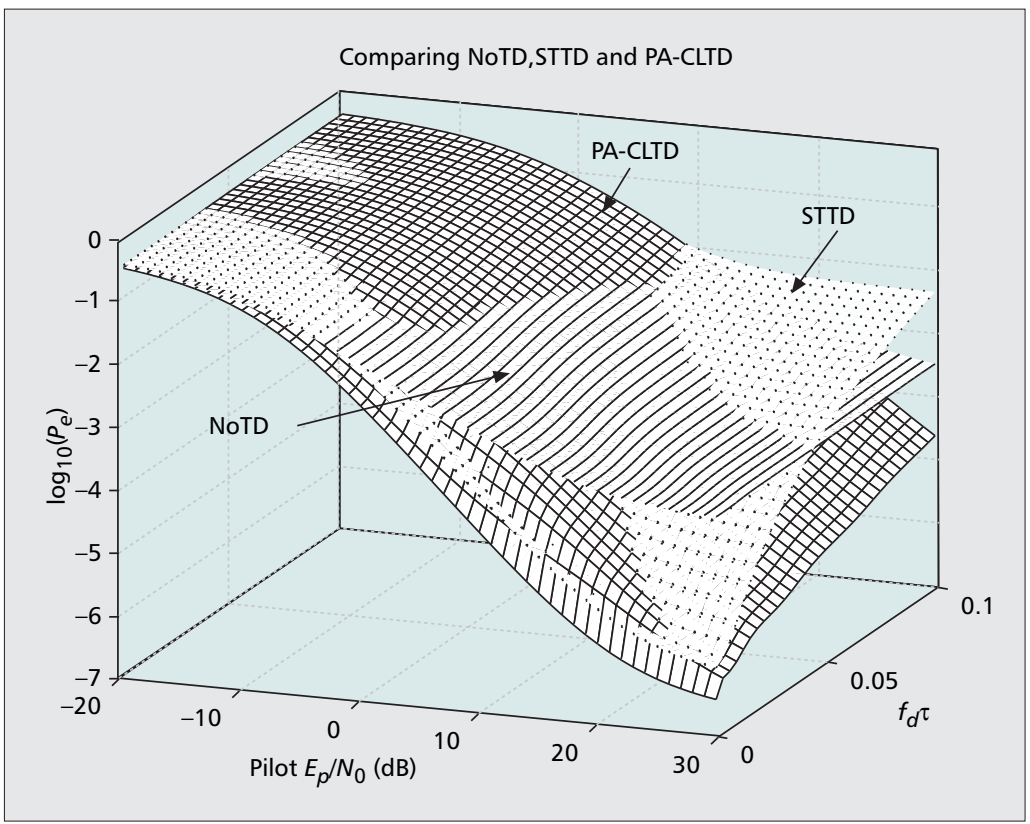

Figure 3. Comparison between SISO (NoTD), Alamouti (STTD), and MRTCLTD (PA-CLTD with one-symbol-period feedback delay).

interference cancellation in [42] requires knowledge of the spreading codes of all interferers. Alternatively, other modulation schemes such as FH SS or OFDM, which are less sensitive to power control errors, can be employed.

\section{ChANNEl Estimation IN MOBILE MIMO DS-CDMA NETWORKS}

The seminal work by Cavers [43] is the first paper that thoroughly analyzed channel estimation error in the context of wireless communication systems. In this article, the bit error probability of a SISO system is found when pilot symbols are time-division multiplexed into the signal stream and an FIR filter is used to combine noisy pilot signals to estimate the channel. This allows the effect of pilot SNR and Doppler spread on the CSI accuracy and the effect of CSI accuracy on the system performance to be defined. The CSI analysis was extended to the convolutionally coded system without interleaving by van Nobelen and Taylor in [44]. From these works, it is known that CSI accuracy improves when the pilot SNR increases or the Doppler spread decreases [43], but diversity increases as the interleaving depth or the Doppler spread increases [44]. Therefore, a diversity-estimation performance trade-off as a function of Doppler spread is expected. This trade-off was thoroughly analyzed only recently by Worthen and Stark in [45], where the optimal memory lengths and the error exponent bounds for joint estimation and decoding were derived assuming a block fading channel. The analysis was further extended by Jootar et al. in [46] for a convolutionally coded SISO CDMA system, where the pilot symbols and the data symbols are transmitted in parallel with different orthogonal codes. In this analysis, the pairwise error probability of the coded SISO system was derived with assumptions that are more general and a model that incorporates implementation issues (e.g., filter coefficients for the channel estimator, interleaving depth, signal-to-pilot energy ratio, multipath delay profile, and the Rician $\mathrm{K}$ factor). The analysis has been shown to accurately predict the frame error probability and the bit error probability from Monte Carlo simulations [46].

The exact performance analysis of MIMO systems in the presence of multiple users is quite involved [47]. The impact of channel estimation in the presence of multiple interferers for SIMO systems has been recently studied in [48]. Closedform expressions for signal-to-interference-plusnoise ratio (SINR) distribution and outage probability are obtained for both equal-power and unequal-power interferer scenarios. These expressions allow for convenient evaluation of the effect of channel estimation quality on system performance.

Analyses on the effect of noisy CSI and Doppler spread on the performance of transmit diversity systems, such as an Alamouti space-time coded system or a closed-loop transmit diversity system with channel codes, are limited. Existing papers focus on systems without channel codes. For example, the effect of noisy CSI and Doppler spread on the Alamouti space-time coded system was derived by Jootar et al. in [49], where the authors assumed no channel codes. In addition, the performance of uncoded closed-loop transmit diversity with maximal ratio transmission (MRT) and equal ratio transmission (ERT) was derived by Jootar et al. in [50], where the authors showed that, although ERT closed-loop transmit diversity requires much less feedback information, the performance of this scheme is surprisingly close to the performance of the MRT scheme. Comparison among the results from $[43,49,50]$ in Fig. 3 provides a complete understanding of how the SISO, Alamouti space-time code, and closedloop transmit diversity behave. In Fig. 3, the channels are assumed to be Rayleigh flat fading with the Jakes power spectral density. The orthogonal codes used for the pilot channels and the data channels are assumed to have the same spreading gain. The channel estimates are calculated by 11-tap Wiener filters (noncausal for SISO and the Alamouti space-time code but causal for the MRT-CLTD). From the figure, it can be seen that transmit diversity schemes do not always outperform the SISO system, especially when the pilot SNR is small and the Doppler spread is large.

\section{MIMO-FHSS IN MANETS}

Frequency-hopping spread-spectrum (FHSS) is less sensitive to the near-far problem than DSCDMA, because strong interferers normally collide with the desired user in frequency only for a small fraction of time due to hopping. This makes FHSS attractive for decentralized MANET applications where accurate power control is difficult to achieve [51].

Channel estimation is quite different for FHSS waveforms than for direct-sequence spread spectrum (DSSS), especially in the MANET scenario. For example, users in a DSCDMA downlink estimate the channel realization from the pilot transmitted on a code orthogonal to all users' codes [50]. Although 
such a pilot channel can be implemented in FHSS by reserving a hopping pattern, it is not easily shared by uncoordinated users, since two channels are uncorrelated if the carrier frequency spacing exceeds the coherence bandwidth of the fading environment. Also, due to frequency separation between hops, the channel estimation may have to be performed for each dwell (the time duration of each frequency hop) separately. Consequently, pilot symbols have to be inserted in every dwell. Although blind or semi-blind techniques can be used to reduce the overhead due to excessive training at the cost of increased complexity [52], it is generally difficult to estimate the channel realizations in FHSS, even for a single transmit/receive antenna. Due to this and other considerations, previous studies on FHSS/FHMA systems mainly focus on FSK modulation with noncoherent demodulation (see, e.g., [53]).

It is well-known that multiple antennas can be used to provide a trade-off between diversity gain and multiplexing gain [54]. As argued in [50], MIMO transceivers designed for coherent operation may be sensitive to inaccurate channel estimates and the gain from multiple antennas can be significantly reduced. Conversely, the differential unitary space-time modulation proposed in $[17,55]$ achieves full diversity from the spatial degrees of freedom without requiring knowledge about the channel realization at the receiver. To reduce the error floor in the performance of conventional differential detection [55], decision-feedback detection is proposed in [56] to exploit the statistics of the channel (see also [57]). As will be discussed in more detail below, the statistics of the channel are more stable than the channel realization. In [58], the statistics of the channel and the noise plus interference are estimated adaptively through a proposed joint-estimation demodulation-decoding scheme. The decoding-error performance is studied by [58] in a coded FHSS system using differential unitary space-time modulation. Simulation results show that the proposed receiver significantly suppresses bursty partial band interference (PBI) and the performance loss due to imperfect estimation of the channel/noise/interference statistics is acceptable.

When FHSS is used for multiple access (FHMA), the receiver proposed in [58] may be potentially extended to suppress multiple-access interference (MAI) in addition to PBI. Other approaches for MAI suppression include optimizing the hopping pattern $[59,60]$. MAI suppression by multiple antennas in FHMA networks is of great importance in understanding the impact of MIMO on FHMA networks and requires further investigation. A further question is how to optimally exploit the available degrees of freedom from the space and frequency jointly. As shown in [7, 61], multiple antennas enable multipacket reception and lead to improved network performance if the MAC protocols are designed to exploit this capability.

\section{OFDM MIMO MANETS}

OFDM is a robust modulation scheme with respect to multipath fading; if the OFDM symbol and cyclic prefix durations are chosen accord- ing to the Doppler and delay spreads of the channel, respectively, the OFDM subcarriers retain their orthogonality and experience flat fading upon reception. Thus, high-data-rate transmission is possible without the need for equalization. OFDMA is an OFDM-based multiple-access system, where different users are allocated different subcarriers within each OFDM symbol. It thus combines the aforementioned advantages of OFDM with the important capability for orthogonal multiple access.

As outlined above, FHSS is an attractive alternative to DS-CDMA, especially in noncentralized environments such as ad hoc networks. Along the same line, FH and OFDMA can be combined (FH-OFDMA), such that subcarriers are assigned to users according to predetermined hopping patterns. A cellular system based on a FH-OFDMA system is described in [62, 63]. The hopping patterns are judiciously designed such that intracell interference is avoided and intercell interference is completely randomized [59]. Channel estimation is performed with the help of pilot patterns in the downlink and pilot symbols inserted in each hopping dwell in the uplink. In [64], a theoretical framework is developed in order to evaluate the bit-error-rate performance of coded modulation in the FHOFDMA downlink. The results reveal that the construction and number of pilot patterns can have a large impact on the performance experienced by different users. Naturally, patterns that scan the time/frequency domain in a "uniform" manner guarantee approximately the same channel-estimation quality for different users, resulting in performance fairness. Channel estimation issues for OFDM are also discussed in $[65,66]$. It is shown in [67] that channel estimation accuracy can be higher when the channel is estimated in the time domain as opposed to the frequency domain.

The design concepts described above carry over to the ad hoc environment. In a best-case scenario, different simultaneously communicating links should be assigned orthogonal patterns. If the total bandwidth demand (number of patterns per link $\times$ number of links) exceeds the number of orthogonal patterns (the pool of orthogonal patterns is naturally limited for a given bandwidth), the interfering patterns should be designed such that each link "sees" approximately the same interfering power on the average from other active links. Regarding channel estimation, a pilot pattern must be reserved for each link, since links are geographically separated and thus the respective channels are independent. If such a reservation is not feasible, one or more reference symbols can be inserted in each dwell or differential modulation can be used, as advocated above.

Since FH is employed to deal with the MAI, multiple antennas (MIMO) can be used for spatial multiplexing in order to increase the throughput. The transmitter can transmit orthogonal pilot patterns from each antenna, such that the channel matrix on a given subcarrier can easily be estimated at the receiver. A variety of detection methods can then be employed to retrieve the transmitted vector of symbols [63].
When determining the appropriateness of different MIMO PHY technologies for use in an ad hoc networking context, it is essential that we consider not only the type of channel information required by the specific algorithm, but also the temporal duration over which this information remains useful. 


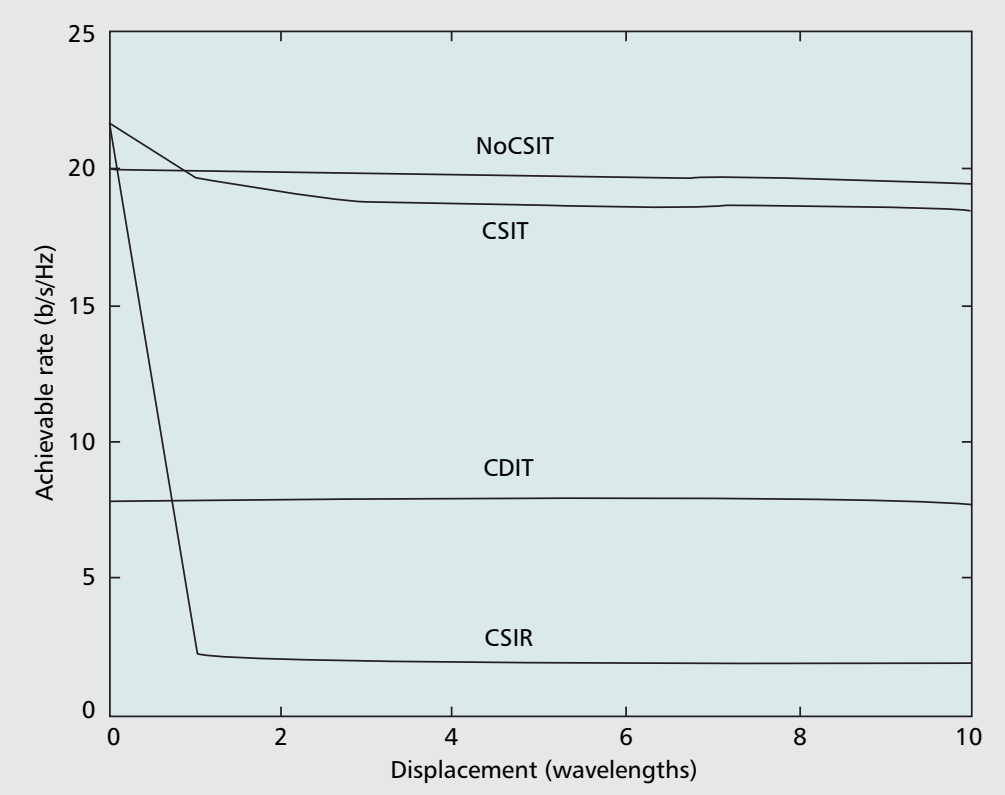

Figure 4. Achievable rate as CSIT, CSIR, CDIT, and NoCSIT go out of date. Results reflect measured data from an outdoor environment with eight transmit and eight receive antennas. study we will use data obtained in a parking lot filled with cars and surrounded by buildings and other structures. Measurements were taken at a carrier frequency of $2.55 \mathrm{GHz}$ and included a stationary transmitter with eight antennas and a mobile receiver traveling at roughly one mile per hour, also with eight antennas. The narrowband channel response was recorded as a function of position along the motion. This channel data allows the implementation of system simulations at any (narrowband) data rate and any node velocity. For more detailed information on the measurement setup, see $[69,70]$.

Capacity and BER for CSI/CDI - For algorithms dependent on CSIT/CSIR, we will assume that perfect CSI is available at some initial point (defined as zero displacement). As the nodes move, the CSI will quickly become outdated, resulting in degraded capacity and bit-error performance. For CDIT, the channel distribution is known at the starting displacement and then slowly goes out of date due to mobility. CSI realizations are drawn directly from the channelmeasurement dataset (i.e., no simulated channel estimation) while CDI is derived from the CSI values averaged over some fixed window size. For example, in the following numerical analysis, the transmit covariance matrix was averaged over a window of one wavelength in order to calculate CDI. In environments with higher mobility, a smaller window size would be needed due to the nonstationarity of the channel statistics.

Figure 4 plots achievable rate when the various forms of channel knowledge go out of date due to node displacement. This displacement represents the time (or distance) between channel updates regardless of whether this knowledge was obtained through tracking, training, or some other form of channel estimation. The curves in Fig. 4 reflect what occurs when the optimal input covariance matrix for Gaussian signaling derived at zero displacement is used on future channel realizations. For this plot, CSIT indicates computing the capacity using the waterfilling solution $[21,71]$ at zero displacement, and using the obtained channel covariance as the channel changes to calculate achievable rate (which implies outdated CSIT but perfect CSIR). The curve for NoCSIT is the uninformed transmit capacity with perfect CSIR. The curve for CSIR is the water-filling solution when the CSI at both the transmitter and receiver becomes outdated. Achievable rate under degraded CSIR is found by assuming that self-interference due to inaccurate channel knowledge is Gaussian [70]. Finally, the curve for CDIT represents the achievable rate based upon beamforming with the dominant eigenvector of the channel transmit correlation matrix. It is important to recognize that this does not generally represent the optimal capacity obtainable with CDIT [68], but is used here to describe the relative stationarity of this form of channel knowledge. We will be focusing on the variation of this curve with time rather than the absolute capacity level. Note that dynamic channel tracking algorithms [72] can be implemented to mitigate the effects of outdated CSI; however, there is always a lag associated with the channel update process. The above 
results define the sensitivity of achievable rate to this relative update rate, based on experimental measurements.

These results imply that the achievable rate is most sensitive to changes in CSI at the receiver, and significantly less sensitive to changes in CSI at the transmitter. This can be understood by recognizing that in most practical channels, while the channel transfer matrix varies rapidly with position, the channel multipath structure (path gains and angles of departure and arrival) is much more slowly varying. Therefore, even outdated CSIT can indicate the general directions in which power should be transmitted for increasing the power at the receiver. The performance when using outdated CDIT is even less sensitive to node position, since the channel distribution depends only on the slowly varying multipath parameters. The BYU database of channel measurements covers many different environments, from the urban environment described here to indoor office buildings or outdoor forested areas, each having somewhat different absolute time scales of degradation; however, they all exhibit the general achievable rate trends discussed in this section.

Figure 5 shows the uncoded bit-error rate (BER) as a function of node displacement using specific algorithms with BPSK modulation. The curves are for direct serial-to-parallel transmission (V-BLAST), transmitting on the right singular vectors of the outdated channel matrix and decoding with either the pseudo-inverse product of the outdated channel matrix and transmit precoding matrix (SVD1) or decoding with the pseudo-inverse product of the current channel matrix and transmit precoding matrix (SVD2), and a differential BPSK constellation with a beamformer derived from the dominant eigenvector of the transmit channel correlation matrix (BF). For V-BLAST, SVD1, and SVD2, only four of the eight streams were used; therefore, the achievable throughput is higher than that of the beamformer. The V-BLAST and SVD1 simulations allow CSIR and CSIT to go out of date while SVD2 assumes perfect channel information at the receiver and awareness of the outdated CSIT used by the transmitter. The beamformer simulation allows CDIT to change versus displacement.

The performance curves of Fig. 5 reflect trends observed in the achievable rates of Fig. 4: poor knowledge of CSI at the receiver directly translates into poor BER performance (VBLAST and SVD1); outdated CSIT is less sensitive to temporal variations (SVD2); and CDIT with NoCSIR is extremely insensitive to changes in the channel (BF). Further, the results of SVD1 and SVD2 demonstrate the dangers in assuming stationarity of the channel. One could consider SVD2 as the expected performance, assuming a constant channel, while SVD1 is the actual performance resulting from node mobility and infrequent channel updates.

While not explicitly studied in this article, it is also possible to define channel goodness information (CGI), which is merely an $N$-bit quantization of a number which defines the overall channel quality. For example, CGI could represent a quantized version of the channel matrix

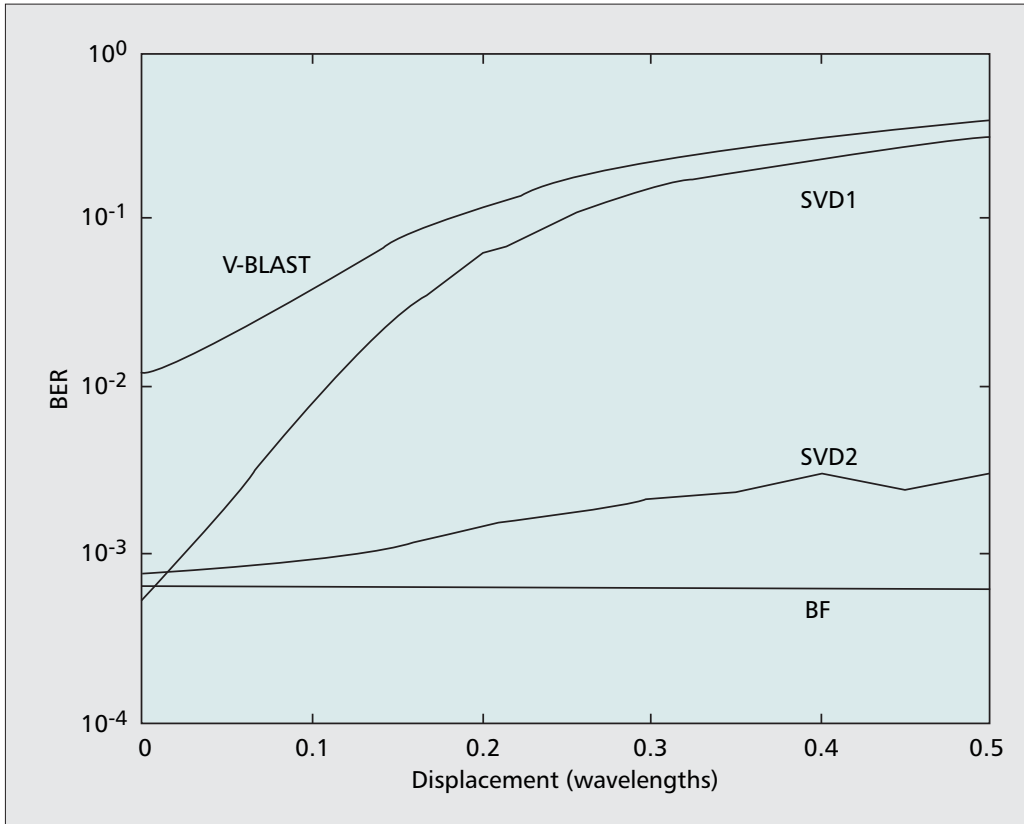

Figure 5. BER for V-BLAST, Covariance Beamforming (BF), and SVD transmission vs. receive node displacement in an $8 \times 8$ MIMO system.

Frobenius norm or even the number of channel singular values that are larger than a specific threshold. Under some circumstances, such CGI can change more slowly than any of the other channel descriptions discussed here, although additional research is required to carefully quantify the variations of such CGI quantities and understand their use in PHY algorithm and networking-layer protocol design.

Multi-User Systems - The observations made above for point-to-point links carry over into spatial processing for multi-user systems. For example, techniques such as dirty-paper coding [73], beamforming, and zero-forcing [23] require CSIT for all links that the transmitter is establishing. Because these algorithms use the CSIT to perform spatial nulling, they will most certainly be highly sensitive to time variation in the CSIT. One interesting possibility, however, is the use of CDIT in a multi-user system such that the transmitter achieves spatial-division multiple access by simply reducing the average interference power at each node. The strength of such an approach is that the CDIT would vary much more slowly with node motion, and therefore would not need to be updated as rapidly. The specific implementation and performance of such a scheme, however, remains an area of future research.

\section{CONCLUSION}

In this article, we have discussed research and design issues related to protocols for ad hoc networks in which nodes are equipped with multiple antennas and the processing capabilities to exploit various forms of MIMO techniques. The main focus was on MAC-layer design via a crosslayer approach in which the specific characteristics of the MIMO PHY are an integral part of the design process. A number of issues need to be taken into account in this case, and have been 
The cross-layer approach invoked in this article, while being very promising for high-performance MIMO ad hoc networks, still requires much research in order to fully understand the many issues involved and successfully exploit the properties of $M I M O$ in this environment. discussed in the article. A specific example of a MAC based on these principles has been given, which highlights the benefits of tying the protocol design and operation to the physical-layer behavior. Finally, the issue of channel estimation, on which the success of MIMO techniques often critically depends, has been reviewed and discussed from the networking protocol design point of view. In doing so, we have tried to point out some important trade-offs related to channel-estimation accuracy, waveform selection, time scales, overhead, and performance.

The cross-layer approach invoked in this article, while being very promising for high-performance MIMO ad hoc networks, still requires much research in order to fully understand the many issues involved and successfully exploit the properties of MIMO in this environment, while not losing some of the architectural advantages that are naturally present in a traditional layered solution. Our goal in this article has been to examine many of these issues and directions as a starting point towards addressing the many challenging problems that remain unsolved.

\section{ACKNOWLEDGMENTS}

This work has been partially supported by the U.S. Army Research Office under Multi-University Research Initiative (MURI) grant no. W91 1NF-04-1-0224. The authors also gratefully acknowledge the useful discussions with and contributions of P. Casari, M. Levorato, H. Sui, J. Jootar, R. Cruz, K. Stamatiou, A. Jagannatham, J. Zheng, E. Gelal, and G. Jakllari.

\section{REFERENCES}

[1] J.H. Winters, "Optimum Combining in Digital Mobile Radio with Co-Channel Interference," IEEE JSAC, July 1984, pp. 528-39.

[2] J. H. Winters, J. Salz, and R. D. Gitlin, "The Impact of Antenna Diversity on the Capacity of Wireless Systems," IEEE Trans. Commun., vol. 42, Feb.-Apr. 1999, pp. 1740-51.

[3] G. J. Foschini, "Layered Space-Time Architecture for Wireless Communication in a Fading Environment when Using Multiple Antennas," Bell Labs Tech. J., vol. 1, 1996, pp. 41-59.

[4] G. Anastasi, M. Conti, and E. Gregori, IEEE 802.11 Ad Hoc Networks: Protocols, Performance and Open Issues, New York: IEEE Press-Wiley, 2004.

[5] S. Gupta and P. R. Kumar, "The Capacity of Wireless Networks," IEEE Trans. Info. Theory, vol. 46, Mar. 2000, pp. 388-404.

[6] S. Krishnamurthy and P. Krishnamurthy, "Use of Smart Antennas in Ad Hoc Networks," Ad Hoc Networks Technologies and Protocols, Springer Science, 2005.

[7] K. Sundaresan et al., "Medium Access Control in Ad Hoc Networks with MIMO Links: Optimization Considerations and Algorithms," IEEE Trans. Mobile Comp., vol. 3, Oct.-Dec. 2004, pp. 350-65.

[8] P. Casari, M. Levorato, and M. Zorzi, "On the Implications of Layered Space-Time Multiuser Detection on the Design of MAC Protocols for Ad Hoc Networks," Proc. IEEE PIMRC, Berlin, Germany, 11-14 Sept. 2005.

[9] V. Kawadia and P. R. Kumar, "A Cautionary Perspective on Cross-Layer Design," IEEE Wireless Commun., vol. 12 , no. 1, Feb. 2005, pp. 4-11.

[10] A. J. Paulraj et al., "An Overview of MIMO Communications: A Key to Gigabit Wireless," Proc. IEEE, vol. 92, Feb. 2004, pp. 198-218.

[11] K. K. Wong, R. Murch, and K. Letaief, "Performance Enhancement of Multiuser MIMO Wireless Communication Systems," IEEE Trans. Commun., vol. 50, Dec. 2002, pp. 1960-70.

[12] Q. Spencer, A. Swindlehurst, and M. Haardt, "ZeroForcing Methods for Downlink Spatial Multiplexing in Multi-User MIMO Channels," IEEE Trans. Sig. Processing, vol. 52, Feb. 2004, pp. 461-71.
[13] Q. Spencer et al., "An Introduction to the Multi-User MIMO Downlink," IEEE Commun. Mag., vol. 42, Oct. 2004, pp. 60-67.

[14] M. Juntti et al., "MIMO MC-CDMA Communications for Future Cellular Systems," IEEE Commun. Mag., vol. 43, Feb. 2005, pp. 118-24.

[15] G. Stuber et al., "Broadband MIMO-OFDM Wireless Communications," Proc. IEEE 2004, vol. 92, Feb. 2004, pp. 271-94.

[16] H. Yang, "A Road to Future Broadband Wireless Access: MIMO-OFDM-Based Air Interface," IEEE Trans. Commun., vol. 43, Jan. 2005, pp. 53-60.

[17] H. Jafarkhani, Space-Time Coding, Academic Press, 2005.

[18] J. Jootar, J. R. Zeidler, and J. F. Diouris, "Performance of Polarization Diversity in Correlated Nakagami-m Fading Channels," IEEE Trans. Sig. Processing, Jan. 2006, pp. 128-36.

[19] D. Palomar and M. Lagunas, "Joint Transmit-Receive Space-Time Equalization in Spatially Correlated MIMO Channels: A Beamforming Approach," IEEE JSAC, vol. 21, June 2003, pp. 730-43.

[20] P. W. Wolniansky et al., "V-BLAST: An Architecture for Realizing Very High Data Rates over the Rich-Scattering Wireless Channel," Proc. URSI ISSSE '98, Pisa, Italy, Sept. 29-Oct. 2, 1998, pp. 295-300.

[21] G. Raleigh and J. Cioffi, "Spatio-Temporal Coding for Wireless Communication," IEEE Trans. Commun., vol. 46, Mar. 1998, pp. 357-66.

[22] T. Taiwen and R. Heath, "Space-Time Interference Cancellation in MIMO-OFDM Systems," IEEE Trans. Sig. Processing, vol. 54, Sept 2005, pp. 1802-16.

[23] Q. Spencer et al. "Performance of Multi-User Spatial Multiplexing with Measured Channel Data" (to appear).

[24] M. Carvalho and J. J. Garcia-Luna-Aceves, "Modeling Wireless Ad Hoc Networks with Directional Antennas," Proc. IEEE INFOCOM, Apr. 2006.

[25] S. Vasudevan, J. Kurose, and D. Towsley, "On Neighbor Discovery in Wireless Networks with Directional Antennas," Proc. IEEE INFOCOM, 2005, vol. 4, pp. 2502-12.

[26] G. Jakllari, W. Luo, and S. V. Krishnamurthy, "An Integrated Neighbor Discovery and MAC Protocol for Ad Hoc Networks Using Directional Antennas," Proc. IEEE WoWMoM, Taormina, Italy, 2005, pp. 11-21.

[27] P. Santi, Topology Control in Wireless Ad Hoc and Sensor Networks, Wiley, 2005.

[28] T. Holliday and A. Goldsmith, "Joint Source and Channel Coding for MIMO Systems," Proc. Allerton Conf. Commun., Control, and Computing, Monticello, IL, Oct. 2004, pp. 1302-11.

[29] M. Kobayashi, G. Caire, and D. Gesbert, "Opportunistic Beamforming vs. Space-Time Coding in a Queued Downlink," Proc. 1ST Summit, 2005.

[30] S. Sfar, R. D. Murch, and K. B. Letaief, "Layered SpaceTime Multiuser Detection over Wireless Uplink Systems," IEEE Trans. Wireless Commun., vol. 2, July 2003, pp. 653-68.

[31] ns-2 simulator, http://www.isi.edu/nsnam/ns

[32] M. Levorato et al., "An Approximate Approach for Layered Space-Time Multiuser Detection Performance and Its Application to MIMO Ad Hoc Networks," Proc. IEEE ICC, Istanbul, Turkey, 11-15 June 2006.

[33] M. Dong and L. Tong, "Optimal Design and Placement of Pilot Symbols for Channel Estimation," IEEE Trans. Signal Proc., vol. 50, Dec. 2002, pp. 3055-69.

[34] B. Hassibi and B. M. Hochwald, "How Much Training Is Needed in Multiple-Antenna Wireless Links?" IEEE Trans. Info. Theory, vol. 49, Apr. 2003, pp. 951-63.

[35] B. Sadler, R. Kozick, and T. Moure, "Bounds on MIMO Channel Estimation and Equalization with Side Information," Proc. IEEE Conf. Acoustics, Speech and Sig. Processing, May 2001, pp. 2145-48.

[36] Y. Sung et al., Training for MIMO Communications, Space-Time Wireless Systems: From Array Processing to MIMO Communications, Cambridge Univ. Press, 2006.

[37] J. Zheng and B. D. Rao, "LDPC-Coded MIMO Systems with Unknown Block Fading Channels: Soft MIMO Detector Design, Channel Estimation, and Code Optimization," IEEE Trans. Sig. Processing, vol. 54, 2006, pp. 1504-18.

[38] S. M. Alamouti, "A Simple Transmit Diversity Technique for Wireless Communications," IEEE JSAC, vol. 16, pp. 1451-58, Oct. 1998

[39] N. Kong and L. B. Milstein, "Error Probability of Multicell CDMA over Frequency Selective Fading Channels with Power Control Error," IEEE Trans. Commun., Apr. 1999, pp. 608-17. 
[40] J. M. Romero-Jerez, C. T'Ellez-Labao, and A. DíazEstrella, "Effect of Power Control Imperfections on the Reverse Link of Cellular CDMA Networks under Multi path Fading," IEEE Trans. Sig. Processing, Jan. 2004, pp. 61-71.

[41] U. Madhow, "Blind Adaptive Interference Suppression for Direct-Sequence CDMA," Proc. IEEE, Oct. 1998, pp. 2049-69.

[42] J. Holtzman and P. Patel, "Analysis of a Simple Successive Interference Cancellation Scheme in a DS/CDMA System," IEEE JSAC, June 1994, pp. 796-807.

[43] J. K. Cavers, "An Analysis of Pilot Symbol Assisted Modulation for Rayleigh Fading Channels," IEEE Trans. Sig. Processing, vol. 40, Nov. 1991, pp. 686-93.

[44] R. V. Nobelen and D. R Taylor, "Analysis of the Pairwise Error Probability of Noninterleaved Codes on the Rayleigh-Fading Channel," IEEE Trans. Commun., vol. 44, Apr. 1996, pp. 456-63.

[45] A. R Worthen and W. E. Stark, "On the Channel Memory-Diversity Trade-off in Communication Systems," Information, Coding and Mathematics, M. Blaum, P. G Farrell, and H. C. A. van Tilborg, Eds., Kluwer, 2002.

[46] J. Jootar, J. R. Zeidler, and J. G. Proakis, "Performance of Convolutional Codes with Finite-Depth Interleaving and Noisy Channel Estimates," IEEE Trans. Commun (to appear).

[47] A. Shah and A. M. Haimovich, "Performance Analysis of Maximal Ratio Combining and Comparison with Optimum Combining for Mobile Radio Communication with Co-Channel Interference," IEEE Trans. Sig. Processing, vol. 49, July 2000, pp. 1454-63.

[48] Y. Tokgoz and B. D. Rao, "The Effect of Imperfect Channel Estimation on the Performance of Maximum Ratio Combining in the Presence of Co-Channel Interference," IEEE Trans. Sig. Processing (to appear).

[49] J. Jootar, J. R. Zeidler, and J. G. Proakis, "Performance of Alamouti Space-Time Code in Time-Varying Channels with Noisy Channel Estimates," Proc. Wireless Commun. and Networking Conf., Mar. 2005, pp. 498-503.

[50] J. Jootar, J. R. Zeidler, and J. G. Proakis, "On the Performance of Closed-Loop Transmit Diversity with Noisy Channel Estimates," Proc. ICC '06, June 2006.

[51] A. Ephremides, J. E. Wieselthier, and D. J. Baker, "A Design Concept for Reliable Mobile Radio Networks with Frequency Hopping Signaling," Proc. IEEE, Jan 1987

[52] A. K. Jagannatham and B. D. Rao, "Whitening-Rotation Based Semi-Blind MIMO Channel Estimation," IEEE Trans. Sig. Processing (to appear).

[53] K. Choi and K. Cheun, "Maximum Throughput of FHSS Multiple-Access Networks Using MFSK Modulation," IEEE Trans. Commun., Mar. 2004, pp. 426-34.

[54] L. Zheng and D. Tse, "Diversity and Multiplexing: A Fundamental Trade-off in Multiple-Antenna Channels," IEEE Trans. Info. Theory, vol. 49, May 2003, pp. 1073-96.

[55] B. M. Hochwald and W. Sweldens, "Differential Unitary Space-Time Modulation," IEEE Trans. Commun. vol. 48, Dec. 2000, pp. 2041-52.

[56] R. Schober and L. Lampe, "Noncoherent Receivers for Differential Space-Time Modulation," IEEE Trans. Commun., vol. 50, May 2002, pp. 768-77.

[57] H. Sui and J. R. Zeidler, "An Explicit and Unified Error Probability Analysis of Two Detection Schemes for Differential Unitary Space-Time Modulation," Proc. 39th Asilomar Conf. Signals, Syst., and Computers, Nov. 2005, pp. 1579-83.

[58] H. Sui and J. R. Zeidler, "Erasure Insertion for Coded DUSTM-FHSS Systems without A Priori Knowledge," Proc. IEEE ICC '06, June 2006.

[59] G. J. Pottie and A. R. Calderbank, "Channel Coding Strategies for Cellular Radio," IEEE Trans. Sig. Processing, vol. 44, Nov. 1995, pp. 763-70.

[60] D. Sarwate, "Optimum PN Sequences for CDMA Systems," Proc. IEEE ISSSTA, 1994, pp. 27-35.

[61] M. Park, R. W. Heath Jr., and S. M. Nettles, "Improving Throughput and Fairness for MIMO Ad Hoc Networks Using Antenna Selection Diversity," Proc. IEEE GLOBECOM, 2004

[62] R. Laroia, S. Uppala, and J. Li, "Designing a Mobile Broadband Wireless Access Network," IEEE Sig. Proc. Sept. 2004, pp. 20-29.

[63] D. Tse and P. Viswanath, Fundamentals of Wireless Communication, Cambridge Univ. Press, 2005

[64] K. Stamatiou and J. G. Proakis, "A Performance Analysis of Coded Frequency-Hopped OFDMA with Pilot Assisted Channel Estimation," Proc. PIMRC, Sept. 2005.
[65] Y. Li, L. J. Cimini, and N. R. Sollenberger, "Robust Channel Estimation for OFDM Systems with Rapid Dispersive Fading Channels," IEEE Trans. Commun., vol. 46, July 1998, pp. 902-15.

[66] Y. Li, "Pilot-Symbol-Aided Channel Estimation for OFDM in Wireless Systems," IEEE Trans. Sig. Processing, vol. 49, July 2000, pp. 1207-15.

[67] A. K. Jagannatham and B. D. Rao, "Complex Constrained CRB and Its Applications to Semi-Blind MIMO and OFDM Channel Estimation," Proc. 3rd IEEE Sensor Array and Multichannel Sig. Processing Wksp., 2004, pp. 397-401.

[68] A. Goldsmith et al., "Capacity Limits of MIMO Channels," IEEE JSAC, vol. 21, June 2003, pp. 684-702.

[69] J. W. Wallace et al., "Experimental Characterization of the MIMO Wireless Channel: Data Acquisition and Analysis," IEEE Trans. Wireless Commun., vol. 2, Mar. 2003, pp. 335-43.

[70] M. A. Jensen and J. W. Wallace, "Recent Advances in Antennas and Propagation for MIMO Systems: MultiUser Networks and Channel Temporal Variation," Proc. 2005 Int'l. Conf. Electromagnetics in Advanced Apps., Sept. 2005.

[71] I. E. Telatar, "Capacity of Multi-Antenna Gaussian Channels," Euro. Trans. Telecommun., vol. 10, Nov. 1999, pp. 585-95.

[72] K. Huber and S. Haykin, "Improved Bayesian MIMO Channel Tracking for Wireless Communications: Incorporating a Dynamical Channel," IEEE Trans. Wireless Commun (to appear)

[73] M. H. M. Costa, "Writing on Dirty Paper," IEEE Trans. Info. Theory, vol. 29, May 1983, pp. 439-41.

\section{BIOGRAPHIES}

MICHELE ZORZI [S'89, M'95, SM'98] (zorzi@ing.unife.it) received Laurea and Ph.D. degrees in electrical engineering from the University of Padova, Italy, in 1990 and 1994, respectively. During academic year 1992-1993 he was on leave at the University of California at San Diego (UCSD), attending graduate courses and doing research on multiple access in mobile radio networks. In 1993 he joined the faculty of the Dipartimento di Elettronica e Informazione, Politecnico di Milano, Italy. After spending three years with the Center for Wireless Communications at UCSD, in 1998 he joined the School of Engineering of the University of Ferrara, Italy, and in 2003 joined the Department of Information Engineering of the University of Padova, Italy, where he is currently a professor. His present research interests include performance evaluation in mobile communications systems, random access in mobile radio networks, ad hoc and sensor networks, protocol design for MIMO networks, and energy-constrained communications protocols. From 2003 to 2005 he was Editor-In-Chief of IEEE Wireless Communications, and currently serves on the Editorial Boards of IEEE Transactions on Communications, IEEE Transactions on Wireless Communications, IEEE Transactions on Mobile Computing, the Wiley Journal of Wireless Communications and Mobile Computing, and the ACM/URSI/Kluwer Journal of Wireless Networks. He has also been a guest editor for special issues of IEEE Personal Communications (Energy Management in Personal Communications Systems) and IEEE Journal on Selected Areas in Communications (Multimedia Network Radios).

JAMES ZEIDLER [ $\left.\mathrm{M}^{\prime} 76, \mathrm{SM}^{\prime} 84, \mathrm{~F}^{\prime} 94\right]$ is a research scientist and senior lecturer in the Department of Electrical Engineering at UCSD. He is a faculty member of the UCSD Center for Wireless Communications and the University of California Institute for Telecommunications and Information Technology. He has more than 200 technical publications and 13 patents in communications, signal processing, data compression techniques, and electronic devices. He was elected Fellow of the IEEE in 1994 for his technical contributions to adaptive signal processing and its applications. He received the IEEE Communications Society Frederick Ellersick Prize at the IEEE Military Communications Conference in 1995, the Navy Meritorious Civilian Service Award in 1991, and the Lauritsen-Bennett Award for Achievement in Science from the Space and Naval Warfare Systems Center in 2000. He was an Associate Editor of IEEE Transactions on Signal Processing.

ADAM L. ANDERSON [S'00] received B.S. (2002) and MS. (2004) degrees in electrical engineering from Brigham Young University, Provo, UT, and is currently working toward a Ph.D. degree in electrical engineering at UCSD. 
His current research interests focus on MIMO systems in mobile ad hoc networks.

A. LeE SWINDLEHURST [S'83, M'84, SM'99, F'04] received B.S. (summa cum laude) and M.S. degrees in electrical engineering from Brigham Young University in 1985 and 1986, respectively, and a Ph.D. degree in electrical engineering from Stanford University in 1991. From 1983 to 1984 he was employed with Eyring Research Institute, Provo, UT, as a scientific programmer. During 1984-1986 he was a research assistant in the Department of Electrical Engineering at Brigham Young University, working on various problems in signal processing and estimation theory. He was awarded an Office of Naval Research Graduate Fellowship for 1985-1988, and during most of that time was affiliated with the Information Systems Laboratory at Stanford University. From 1986 to 1990 he was also employed at ESL, Inc., Sunnyvale, CA, where he was involved in the design of algorithms and architectures for several radar and sonar signal processing systems. He joined the faculty of the Department of Electrical and Computer Engineering at Brigham Young University in 1990, where he holds the position of full professor and is currently serving as department chair. During 1996-1997 he held a joint appointment as a visiting scholar at both Uppsala University, Sweden, and the Royal Institute of Technology, Stockholm, Sweden. His research interests include sensor array signal processing for radar and wireless communications, detection and estimation theory, and system identification, and he has over 140 publications in these areas. He is a past Secretary of the IEEE Signal Processing Society. He is currently serving as a member of the Sensor Array and Multichannel Signal Processing Technical Committee in the same society, as a member of the Editorial Board for EURASIP Journal on Wireless Communications and Networking and IEEE Signal Processing, and is a past Associate Editor for IEEE Transactions on Signal Processing. He was Technical Program Chair for the 1998 IEEE Digital Signal Processing Workshop and the 2001 IEEE International Conference on Acoustics, Speech, and Signal Processing. He is a recipient of severa paper awards, including the 2000 IEEE W. R. G. Baker Prize Paper Award, the 2006 IEEE Signal Processing Society's Best Paper Award, the 2006 IEEE Communications Society Stephen O. Rice Prize in the Field of Communication Theory, and the IEEE Signal Processing Society Young Author Best Paper Award in 2001

Michael A. JENSEN [S'93, M'95, SM'01] received B.S (summa cum laude) and MS. degrees in electrical engineering from Brigham Young University in 1990 and 1991, respectively, and a Ph.D. in electrical engineering at the University of California, Los Angeles (UCLA) in 1994 From 1989 to 1991 he was a graduate research assistant with the Lasers and Optics Laboratory at Brigham Young University. In 1990 he received a National Science Foundation Graduate Fellowship. From 1991 to 1994 he was a graduate student researcher in the Antenna Laboratory at UCLA. Since 1994 he has been at the Electrical and Computer Engineering Department at Brigham Young University, where he is currently the Ward Professor. His main research interests include antennas and propagation, MIMO wireless communications, numerical electromagnetics, and optical fiber communications. He is a member of Eta Kappa Nu and Tau Beta Pi. He currently serves as a member of the Administrative and Joint Meetings Committees for the IEEE Antennas and Propagation Society and has served the society as Vice-Chair and Technical Program Chair for several symposia. He was awarded the H. A. Wheeler paper award in IEEE Transactions on Anten- nas and Propagation in 2002, and best student pape award at the 1994 IEEE International Symposium on Antennas and Propagation.

SRIKANTH V. KRISHNAMURTHY [S'94, M'00] received his Ph.D. degree in electrical and computer engineering from UCSD in 1997. From 1998 to 2000 he was a research staff scientist at the Information Sciences Laboratory, HRL Laboratories, LLC, Malibu, CA. Currently, he is an associate professor of Computer Science at the University of California, Riverside. His research interests span satellite and wireless networks. He has been a principal investigator or project lead on projects from various DARPA programs, including the Fault Tolerant Networks program, the Next Generation Internet program, and the Small Unit Operations program. $\mathrm{He}$ is the recipient of the NSF CAREER Award from ANI in 2003. He also co-edited the book Ad Hoc Networks: Technologies and Protocols (Springer Verlag, 2004). He is the Associate Editor-in-Chief of ACM MC2R.

BHASKAR D. RAO [S'80, M'83, SM'91, F'00] received his B.Tech. degree in electronics and electrical communication engineering from the Indian Institute of Technology, Kharagpur, in 1979, and MS. and Ph.D. degrees from the University of Southern California in 1981 and 1983, respectively. Since 1983, he has been with UCSD, where he is currently a professor in the Electrical and Computer Engineering Department. He has been a member of the IEEE Signal Processing Theory and Methods Technical Committee. He is currently a member of the Signal Processing for Communication Technical Committee and also serves on the EURASIP Signal Processing Journal editorial board. His interests are in the areas of digital signal processing, estimation theory, and optimization theory, with applications to digital communications, speech signal processing, and human-computer interactions.

John PRoAKIS [S'58, M'62, F'84, LF'99] received a B.S.E.E. degree from the University of Cincinnati in 1959, an M.S.E.E. degree from Massachusetts Institute of Technology in 1961, and a Ph.D. degree from Harvard University in 1967. He is an adjunct professor at UCSD and Professor Emeritus at Northeastern University. He was a faculty member at Northeastern University from 1969 through 1998 and held the following academic positions: associate professor of electrical engineering, 1969-1976; professor of electrical engineering, 1976-1998; Associate Dean of the College of Engineering and Director of the Graduate School of Engineering, 1982-1984; Interim Dean of the College of Engineering, 1992-1993; and Chair of the Department of Electrical and Computer Engineering, 1984-1997. Prior to joining Northeastern University, he worked at GTE Laboratories and the MIT Lincoln Laboratory. His professional experience and interests are in the general areas of digital communications and digital signal processing. He is the author of the book Digital Communications (McGraw-Hill, 2001, 4th ed.), and co-author of the books Introduction to Digital Signal Processing (Prentice Hall, 2007, 4th ed.); Digital Signal Processing Laboratory (Prentice Hall, 1991); Advanced Digital Signal Processing (Macmillan, 1992); Algorithms for Statistical Signal Processing (Prentice Hall, 2002): Discrete-Time Processing of Speech Signals (Macmillan, 1992; IEEE Press, 2000); Communication Systems Engineering (Prentice Hall, 2002, 2nd ed.): Digital Signal Processing Using MATLAB V.4 (Brooks/Cole-Thomson Learning, 1997, 2000); Contemporary Communication Systems Using MATLAB (Brooks/Cole-Thomson Learning, 1998, 2000); Fundamentals of Communication Systems (Prentice Hall, 2005). 